\title{
Characteristics of the asymmetric Stockbridge damper
}

\author{
Zakhele Zondi ${ }^{1 *}$, Modify Kaunda ${ }^{2}$, and Tiyamike Ngonda $^{2}$ \\ ${ }^{1}$ Mangosuthu University of Technology, Department of Mechanical Engineering, Durban, South \\ Africa \\ ${ }^{2}$ Cape Peninsula University of Technology, Department of Mechanical Engineering, Cape Town, \\ South Africa
}

\begin{abstract}
The Stockbridge damper (tuned mass absorber) is used on overhead transmission power lines to suppress wind-induced vibrations. These power lines get exposed to various types of wind motions that cause them to vibrate and this causes fatigue failure at the suspension clamp where the maximum stress occurs. Aeolian vibration is the most common wind motion that causes fatigue and eventually, failure to transmission lines. This paper presents the study of an asymmetric Stockbridge damper. A set of experiments were conducted on the damper according to the Institute of Electrical and Electronics Engineers (IEEE) 664 standards to evaluate the characteristics of the asymmetric Stockbridge damper. The results obtained revealed that the asymmetric damper is four degrees of freedom. The results also revealed a relationship between the mass and the magnitude of the resonance frequency.
\end{abstract}

\section{Introduction}

Overhead power transmission lines get exposed to wind motions that cause them to vibrate. Wind motions cause vortex shedding and eventually leads to a fatigue failure, usually at the suspension clamp where the most significant stresses of the power lines occur [1]. This phenomenon leads to a power outage. There are three wind-induced mechanical vibrations of power lines, wake-induced oscillation, conductor galloping, and Aeolian vibration [2]. The wake-induced oscillation of a bundle conductor transmission line is caused by the wake interference between sub-conductors [2]. The spacing between sub-conductors of a bundle conductor is usually in the range of 15-20 times the sub-conductor diameter. During vibrations, the sub-conductors vibrate within sub-spans, which leads to clashing and fatigue failure of conductors. In cold climates, the build-up of ice or snow causes the conductor galloping, which is characterised by a low frequency, high amplitude, mainly vertical conductor motion [2,3]. In most cases, ice builds up on the windward surface of the conductor. As a result, the conductor creates an aerodynamically unstable shape that produces large forces and moments on the conductor. The motion of the conductor due to wind can lead to oscillations in a vertical direction. Aeolian vibration is the most common and familiar

\footnotetext{
* Corresponding author: zakhele@,mut.ac.za
} 
wind motion that affects power lines and produces fatigue failure. It is characterised by a frequency ranging from 3 to $150 \mathrm{~Hz}$ produced by a wind speed of $1 \mathrm{~m} / \mathrm{s}$ to $7 \mathrm{~m} / \mathrm{s}$ [4].

Various researchers have investigated measures to minimise failure of power lines arising from wind induced conductor vibrations. They reported on different designs of wind motion vibration dampers that can be used to mitigate fatigue failure of power lines, one of which is a Stockbridge damper. The main function of a Stockbridge damper is to absorb Aeolian vibration by creating destructive interference of resonance frequencies. Over the past years, there have been several research studies that focused on improving Stockbridge dampers [5]. Barbieri and Barbieri [6] examined the linear and nonlinear dynamical behaviour of asymmetric Stockbridge damper with excitation frequencies in the range of 5 to $17 \mathrm{~Hz}$ using a machine cam with five different profiles. The authors reported that the motion amplitude of vibration is inversely proportional to the natural frequency. Vaja et al. [7] developed a mathematical model with minimum assumptions of the Stockbridge damper that suits the conductor cable. The bending stiffness of the cable and the effect of this stiffness on the natural frequencies were examined. The authors found equivalent stiffness compensated for the assumption of modeling the messenger. The results obtained revealed that the moment of inertia of the power line was directly proportional to the inertia of the messenger cable. Vaja et al. [8] developed an analytical model of a fresh vibration damper with an increased number of resonant frequencies. A 3D finite element model was developed to validate the analytical model. Markiewicz [9] presented a method and computational model for the evaluation of the optimum dynamic characteristics of Stockbridge dampers to be mounted near dead! end towers. The method was applied to a general model of a tension system with insulator strings and antiarc protection elements. It revealed how the efficiency of a standard damper used in such spans may be enhanced by its proper location on a cable.

Although several research studies have been conducted to improve the efficiency of the Stockbridge damper, there have been limited research studies that have focused specifically on the asymmetric Stockbridge damper. This paper aims at examining the characteristics of the asymmetric Stockbridge damper to improve the knowledge pool about this damper. This will assist in the future improvements of the asymmetric Stockbridge damper. Experiments will be conducted on different sizes of an asymmetric Stockbridge damper.

\section{Design, functioning, and history of the Stockbridge damper}

Fig. 1 and Fig. 2. Illustrate the types of Stockbridge dampers, namely asymmetric and symmetric dampers respectively. In general, the asymmetric Stockbridge damper (4-R damper) consists of two unequal masses attached to the end of two unequal lengths of stranded cables, which are known as messenger wires, as shown in Fig. 1 [10]. It also consists of a clamp that is used to clamp damper on the transmission line. The symmetric Stockbridge damper (2-R damper) differs from an asymmetric damper with two equal masses and lengths of stranded cables, as shown in Fig. 2.

The symmetric damper is known as the acronym $2-\mathrm{R}$, it is a two-resonance system. The two resonance frequencies produced by the damper are the ones that counteract the resonance frequency produced by the transmission line when is it exposed to wind motion. The asymmetric damper is known as acronym 4-R, the four resonance frequencies produced by the damper counteract the resonance frequencies produced by the transmission line during operation. 


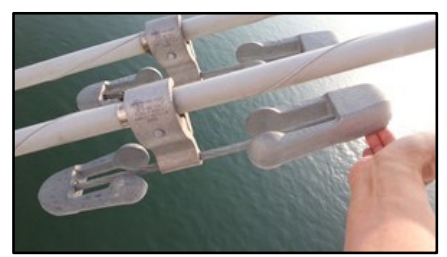

Fig. 1. Asymmetric Stockbridge damper [2].

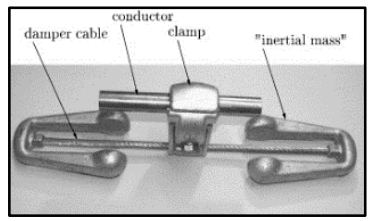

Fig. 2. Symmetric Stockbridge damper [2].

The first Stockbridge-type damper was invented by H. Stockbridge in 1924 after discovering the failure of overhead transmission lines as a result of wind vibrations, as shown in Fig. 3 [2]. Developments and modifications from the original design were conducted and invented to improve the efficiency of the damper. In 1932, Monroe and Templin improved the damper to two degrees of freedom, as shown in Fig. 4 [2]. Both the moment of inertia and the shape were improved to counteract the second vibration mode of the cantilever beam within the frequency range of operation of the damper [2, 10]. In 1968 Claren and Diana came with a new idea in which the two halves of the Stockbridge damper were made asymmetrical leading to two different masses with different moments of inertia and unequal lengths of the messenger cable[2]. A damper known as the Haro damper, as shown in Fig. 5a, was developed by Lauri Haro and Tapani Seppa based on the Stockbridge damper principle in 1970 [2]. An Australian Dogbone damper that develops a torsional resonance was designed in addition to the two flexural resonances, as shown in Fig. 5b. A torsional damper, shown in Fig. 5c, was designed by Tebo of Ontario Hydro and it was mostly used in Canada and other countries worldwide for many years. Elgra is a Swedish damper that consists of a vertical stem having three cast masses loosely fitted to a vertical shaft, as shown in Fig. 5d. The Bretelle damper, shown in Fig. 5e, is widely used in France. It is made from pieces of scrap conductors that are the same size as the line on which it is used [2]. Festoon dampers, shown in Fig. 6, had been used on various long span. Practically no design rules had been published for their use [2].

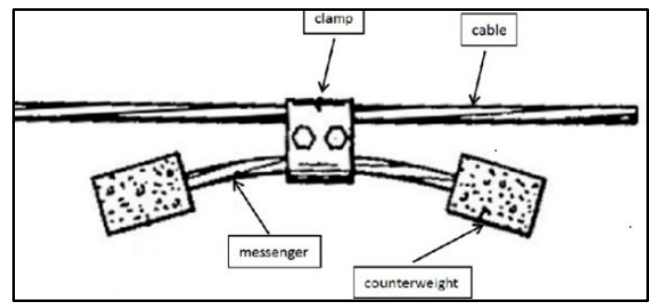

Fig. 3. The original type of Stockbridge damper [2]. 


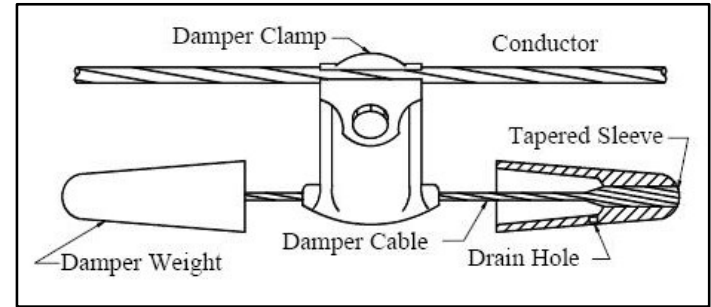

Fig. 4. Monroe and Templin damper [2].

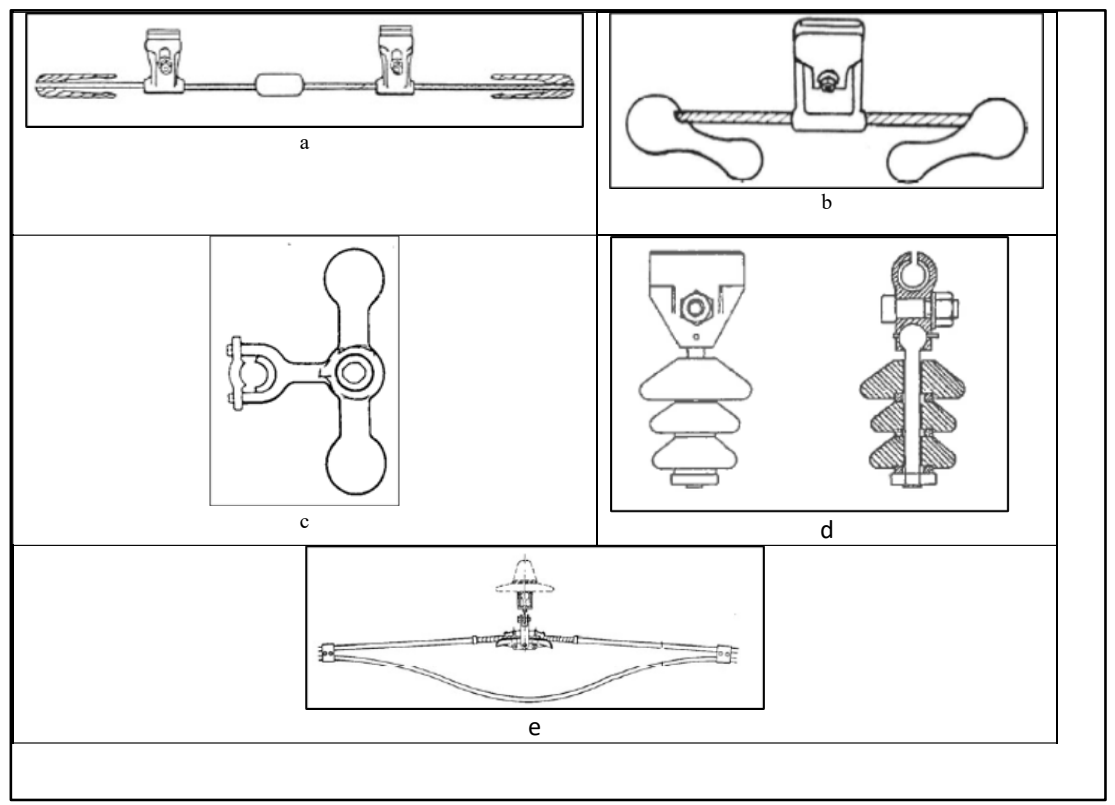

Fig. 5. Different types of dampers: (a) Haro damper. (b) Dogbone damper. (c) torsional damper. (d) Elgra damper. (e) Bretelle damper [2].

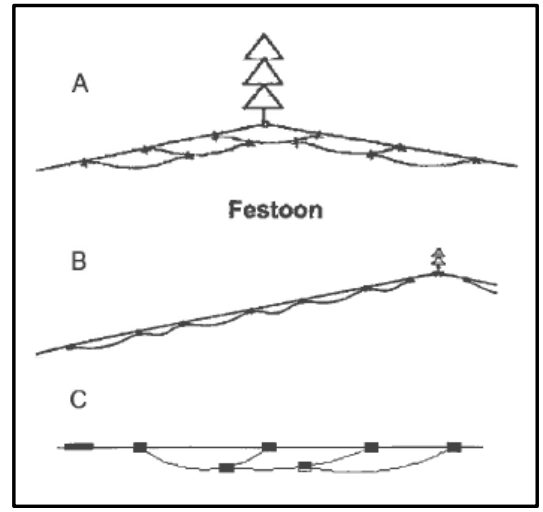

Fig. 6. Festoon dampers. (A) and (B) are festoon dampers for suspension; (C) is a festoon damper for tension points [2]. 


\section{Method}

The equipment used to conduct the experimental work is shown in Fig. 7. The asymmetric Stockbridge damper is not clamped on the power transmission line, it is mounted on the shaker shown in Fig. 8 to represent the transmission line exposed to Aeolian vibration. The speed of the shaker was kept with a constant velocity of $0.1 \mathrm{~m} / \mathrm{s}$ while the frequency was increasing in a range of $5-300 \mathrm{~Hz}$. Three (3) asymmetric Stockbridge dampers of different sizes were tested on a shaker and the test was repeated three times per sample. Two accelerometers were stuck on each damper's mass and one on the shaker base using glue as shown in Fig. 8. The important components of a vibration system consist of:

- An electro-dynamic shaker (TIRA Model, Type TV 56263/LS-340) for providing an input force to the damper.

- An amplifier to control the voltage or current to the shaker.

- A control system (known as a computer-controlled data acquisition system)

- A compressor for load protection

- Three (3) asymmetric Stockbridge dampers of various sizes.

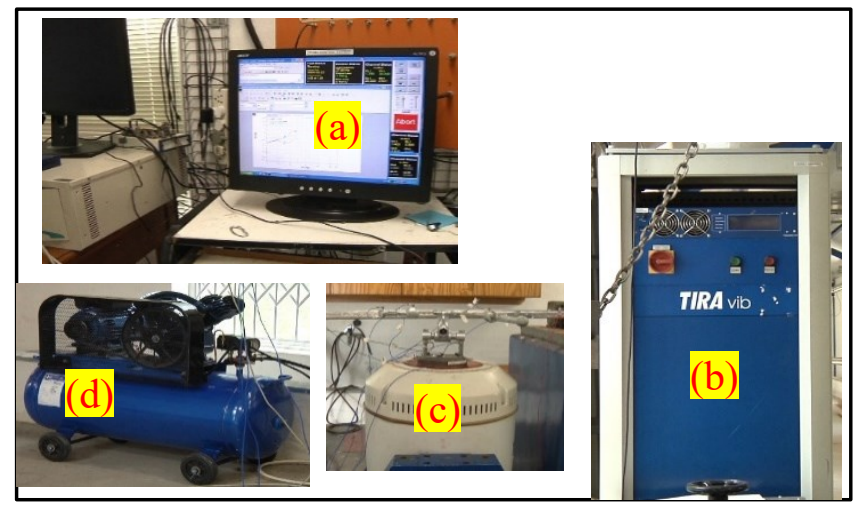

Fig. 7. Components of the vibration test system on the shaker base: (a) controller. (b) Amplifier. (c) Shaker, asymmetric damper, and accelerometers. (d) Compressor.

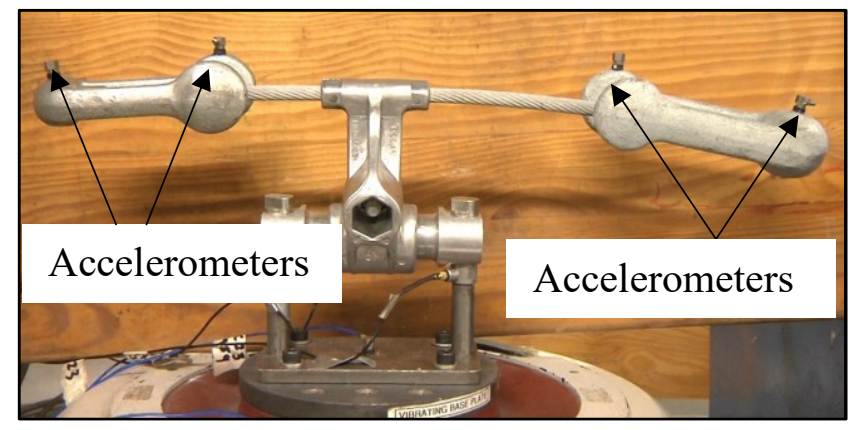

Fig. 8. Stockbridge damper with three accelerometers on each mass [2]. 


\section{Results and discussions}

Fig. 9 below shows the positions at which the resonance frequencies were obtained as per the arrangement of accelerometers. During the operation, the physical observation made on the behaviour of the Stockbridge damper is strictly characterised by a sequential vibration pattern. The outer part $\left(\mathbf{f}_{1}\right)$ of a bigger mass vibrates then translates the vibration signal to the outer part of a smaller mass $\left(\mathbf{f}_{\mathbf{4}}\right)$ as the frequency was increasing. Thereafter the inner part $\left(\mathbf{f}_{2}\right)$ of the bigger mass vibrates which is ultimately followed by the inner part $\left(\mathbf{f}_{3}\right)$ of the smaller mass as the frequency was increasing. Overall, the sequential movement is the one leading the vibration of the damper. The sequence will start from $\mathbf{f}_{\mathbf{1}}, \mathbf{f}_{\mathbf{4}}, \mathbf{f}_{\mathbf{2}}$, and $\mathbf{f}_{\mathbf{3}}$. There are effectively two positions per mass on the Stockbridge damper responsible to produce resonance frequencies, as shown in Fig. 9.

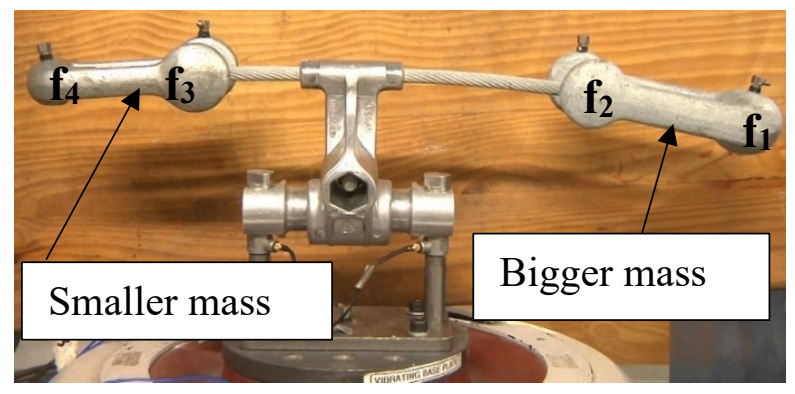

Fig. 9. The asymmetric Stockbridge damper

The graph of acceleration versus frequency shown in Fig. 10 presents the results of a damper (182 025-301) Ø31- $39 \mathrm{~mm}$. From the big mass (at point $\mathbf{f}_{1}$ ), as shown in Fig. 9, it was observed that the frequency of vibration is $8 \mathrm{~Hz}$ with an acceleration amplitude of $0.6 \mathrm{G}$ and $1 \mathrm{G}$ as shown in Fig. 10. From the same big mass (at point $\mathbf{f}_{2}$ ) the frequency of vibration was observed to be $50 \mathrm{~Hz}$ with an acceleration amplitude of $1.9 \mathrm{G}$ and $5 \mathrm{G}$, as shown in Fig. 10. Based on these results the bigger mass has an output of two resonance frequencies and four acceleration amplitudes (inner and outer part). In all three tests conducted, similar results were achieved concerning resonance frequencies and acceleration amplitudes. 


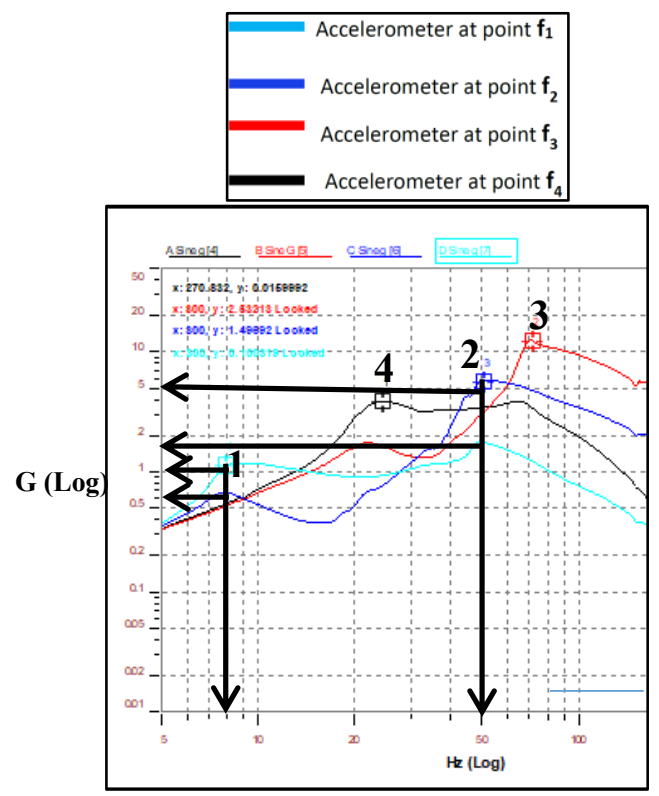

Fig. 10. The graph of acceleration amplitude $\mathrm{G}(\log )$ vs $\mathrm{Hz}(\log )$

Fig. 11 presents the results of a damper (182 025-301) Ø31-39 mm. From the small mass (at point $\mathbf{f}_{4}$ ), as shown in Fig. 9, it was observed that the frequency of vibration is $22 \mathrm{~Hz}$ with an acceleration amplitude of $1.9 \mathrm{G}$ and $4 \mathrm{G}$ as shown in Fig. 11. From the same small mass (at point $\mathbf{f}_{3}$ ), the frequency of vibration was observed to be $70 \mathrm{~Hz}$ with an acceleration amplitude of $4 \mathrm{G}$ and $10 \mathrm{G}$, as shown in Fig. 11. Based on these results the smaller mass has an output of two resonance frequencies and four acceleration amplitudes (inner and outer part). In all three tests conducted, similar results were achieved concerning resonance frequencies and acceleration amplitudes. 


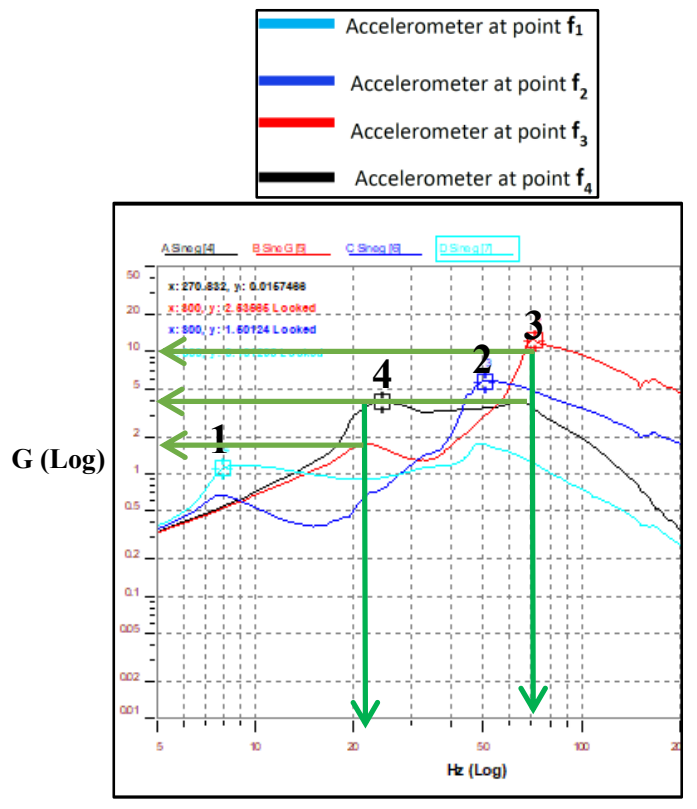

Fig. 11. The graph of acceleration amplitude $\mathrm{G}(\log )$ vs $\mathrm{Hz}(\log )$

At the outer parts of both weights (at points $\mathbf{f}_{\mathbf{1}}$ and $\mathbf{f}_{\mathbf{4}}$ ) the resonance frequencies produced are smaller than the inner parts of the masses (at points $\mathbf{f}_{\mathbf{2}}$ and $\mathbf{f}_{3}$ ). This can be linked to the vibration amplitudes being greater at the outer part of the masses than inner parts, as a result of mass size. Based on the results obtained, the asymmetric Stockbridge damper is four degrees of freedom. The change in the sizes of the asymmetric Stockbridge dampers produces the same number of frequencies and acceleration amplitudes but different magnitudes.

\section{Conclusions}

The experiments were successfully conducted on different sizes of asymmetric Stockbridge dampers. The results revealed that an asymmetric Stockbridge damper can absorb four resonance frequencies. The magnitude of the resonance frequency was inversely proportional to the acceleration amplitudes and the size of the mass. The outer part of the mass produced smaller frequencies than the inner part of the mass due to mass size. The number of resonance frequencies produced by the asymmetric Stockbridge dampers is the same no matter how big or small the asymmetric Stockbridge damper is. At any other resonance frequencies except those $\left(\mathbf{f}_{1}, \mathbf{f}_{2}, \mathbf{f}_{3}\right.$, and $\left.\mathbf{f}_{\mathbf{4}}\right)$ produced by the asymmetric Stockbridge damper, the damper is not a mass absorber. For the future, the work that will be done involves doing experiments when the damper is altered on the transmission line and measured again, in an attempt to see how cable lengths and masses affect the measured response.

\section{Acknowledgment}

This work was supported by the Cape Peninsula University of Technology, Mangosuthu University of Technology, and the VRTC laboratory at UKZN. The authors thank Mr. Pravesh Moodley and Mr. Mthobisi Zulu for their contribution. 


\section{References}

1. O. Griffin, G. Koopmann, "The vortex-excited lift and reaction forces on resonantly vibrating cylinders," Journal of Sound and Vibration, vol. 54, pp. 435-448, (1977)

2. J. Chan, EPRI transmission line reference book: Wind induced-induced conductor motion. Palo Alto, California: Electric Power Research Institute (2006)

3. L. Cloutier, G. Diana, A. Goel, C. Hardy, J. Lilien, J. Wang, "EPRI Transmission Line Reference Book: Wind-Induced Conductor Motion" (2006)

4. A. L. Braun, A. M. Awruch, "Aerodynamic and aeroelastic analyses on the CAARC standard tall building model using numerical simulation," Computers \& Structures, vol. 87, pp. 564-581 (2009)

5. D. Havard, "Assessment of the Cowal JCT x Longwood TS for vibration control," Toronto, Ontario (2008)

6. N. Barbieri, R. Barbieri, "Dynamic analysis of Stockbridge damper," Advances in Acoustics and Vibration, vol. 2012, (2012)

7. N. K. Vaja, O. Barry, B. DeJong, "Finite Element Modeling of Stockbridge Damper and Vibration Analysis: Equivalent Cable Stiffness," in ASME 2017 International Design Engineering Technical Conferences and Computers and Information in Engineering Conference (2017)

8. N. Vaja, O. Barry, E. Tanbour, "On the modeling and analysis of a vibration absorber for overhead powerlines with multiple resonant frequencies," Engineering Structures, vol. 175, pp. 711-720(2018)

9. M. Markiewicz, "Optimum dynamic characteristics of Stockbridge dampers for dead-end spans," Journal of sound and vibration, vol. 188, pp. 243-256, (1995)

10. J. Chan, D. Havard, C. Rawlins, G. Diana, L. Cloutier, J.-L. Lilien, et al., "EPRI Transmission Line Reference Book: wind-induced Conductor Motion” (2009) 